\title{
Seniors and self-harm factor in the opioid crisis
}

Cite as: CMAJ 2017 January 9;189:E42-3. doi: 10.1503/cmaj.109-5357

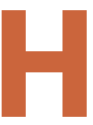
arm from opioids has spiked over the past decade, but the reasons why are likely more complicated than an increase in abuse of the drugs, the Canadian Institute for Health Information (CIHI) warns in a benchmark report.

Thirteen Canadians are hospitalized every day for opioid poisoning, and the rate of hospitalizations has increased more than $30 \%$ since 2007 , according to the report. Seniors aged 65 and older had the highest rates and accounted for nearly a quarter of these hospitalizations, despite representing just $16 \%$ of the Canadian population. And although most hospitalizations related to opioids were due to accidental poisonings, an alarming $33 \%$ overall were intentional - raising new questions about the role of mental health and suicide prevention in curbing opioid deaths.

"When you look at both the hospitalizations and the emergency department visits, many of these are potentially preventable," says Michael Gaucher, CIHI's director of pharmaceuticals and health workforce information. The study provides a baseline for tracking the crisis, although it doesn't capture harms and deaths that occur outside of hospitals, he says. "What we're seeing is potentially underestimating the magnitude of the opioid poisonings."

Gaucher partly attributes the recent surge in hospitalizations to increased use of both prescribed and illegal opioids in Canada, but he says that doesn't account for the full picture. Opioid poisonings among seniors are a case in point, "probably a lot of what would be considered issues related to polypharmacy," he explains.

Seniors consistently had the highest rates of hospitalizations, reaching 20 per 100000 people in 2014-2015. This may be because they are more sensitive to drugs overall and take a higher number of medications than younger adults, increasing their risk of bad reactions, says Dr. David
Lussier, director of the geriatric pain clinic at the McGill University Health Centre in Montréal.

"Also, because pain is more common in older people, they take more opioids, more frequently," he says. But that doesn't necessarily mean they are abusing or being overprescribed opioids. Increases in the senior population and medications mix-ups could also be to blame, Lussier explains.

He was more concerned that $14 \%$ of the opioid hospitalizations among seniors were due to intentional poisonings, defined by $\mathrm{CIHI}$ as "when an opioid is taken with the intent of causing self-harm."

"I am surprised that it is so high," Lussier says. "We therefore have to make sure that we address depression associated with pain in these patients."

Psychological therapies, such as cognitive behavioural therapy, have proven helpful for both pain management and depres-

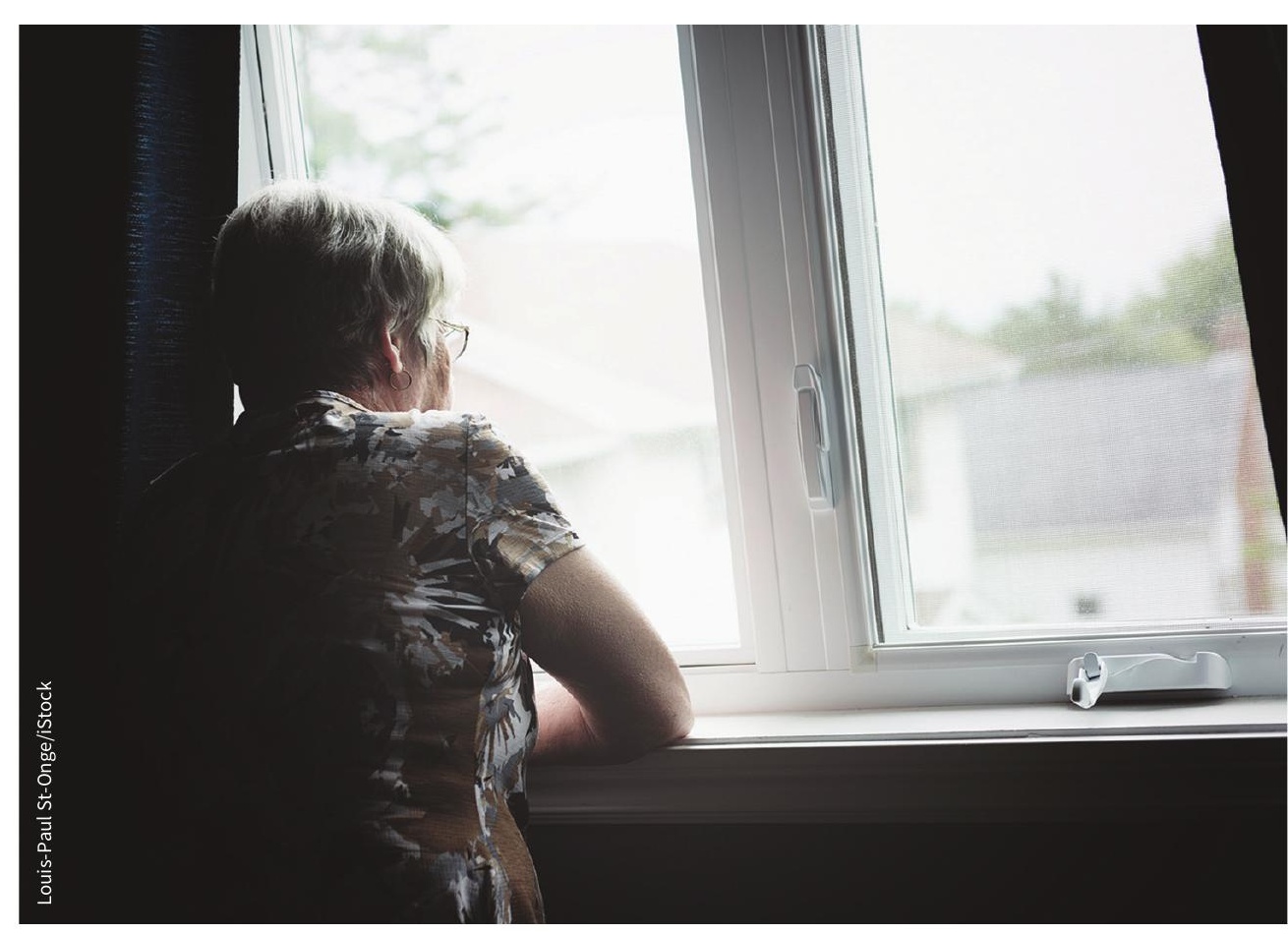

New data show women over 65 have the highest rates of hospitalization for opioid poisoning.

sion, he adds. "The problem is access; most often it's not covered by public insurance so people have to pay out of pocket and that poses an obstacle to treatment."

Improving mental health supports may also help reduce opioid harms among youth aged 15 to 24 . They had the fastest growing rate of hospitalizations, increasing by $62 \%$ to 10 per 100000 people in 2014-2015. More than half of these were for intentional overdoses.

The number of opioid poisonings also differed across the provinces and territories. Rates of hospitalizations ranged from a high of 20.5 per 100000 people in Saskatchewan, closely followed by Alberta and British Columbia, to a low of 9.7 per 100000 people in Quebec. Ontario and Manitoba had rates only slightly higher than Quebec.

Gaucher attributes the "fairly significant" gap between Central and Western 
Canada to a variety of potential factors, including differences in prescribing practices, opioid use and the health of these populations.

According to Lussier, "we know that there is less opioid prescribing in Quebec than in other provinces for sure." That may be because of greater access to psychotherapy, physiotherapy and other non-drug treatments for pain, but "there is no way to know," he says.
Dr. Peter Butt, a substance abuse expert and associate professor of family medicine at the University of Saskatchewan, notes that Western Canada also faces unique challenges from illegal opioids. The availability of illegal fentanyl, an opioid 50 to 100 times more potent than morphine, "moved from west to east as it deposed the heroin trade in $\mathrm{BC}$," he explains.

Increased hospitalizations for opioid poisoning in Western Canada may be a sign that efforts to curb opioid deaths are working, Butt adds. Improved access to antioverdose drugs and take-home kits has increased the number of people who survive poisonings. "Those who survive are often admitted," he says.

$\mathrm{CIHI}$ will follow up the opioid poisonings study with a pan-Canadian report on opioid use in spring 2017.

Lauren Vogel, CMAJ 